\title{
The optimal synthesis of scanned linear antenna arrays
}

\author{
Anas A. Amaireh ${ }^{1}$, Asem S. Al-Zoubi ${ }^{2}$, Nihad I. Dib ${ }^{3}$ \\ 1,2Telecommunications Engineering Department, Yarmouk University, Jordan \\ ${ }^{3}$ Electrical Engineering Department, Jordan University of Science and Technology, Jordan
}

\begin{tabular}{l} 
Article Info \\
\hline Article history: \\
Received Mar 24, 2019 \\
Revised Oct 7, 2019 \\
Accepted Oct 17, 2019 \\
\hline Keywords: \\
Antlion optimization \\
Hybrid optimization algorithm \\
Metaheuristic algorithm \\
Optimization \\
Scanned antenna array
\end{tabular}

\begin{abstract}
In this paper, symmetric scanned linear antenna arrays are synthesized, in order to minimize the side lobe level of the radiation pattern. The feeding current amplitudes are considered as the optimization parameters. Newly proposed optimization algorithms are presented to achieve our target; Antlion Optimization (ALO) and a new hybrid algorithm. Three different examples are illustrated in this paper; 20, 26 and 30 elements scanned linear antenna array. The obtained results prove the effectiveness and the ability of the proposed algorithms to outperform and compete other algorithms like Symbiotic Organisms Search (SOS) and Firefly Algorithm (FA).
\end{abstract}

Copyright $(2020$ Institute of Advanced Engineering and Science. All rights reserved.

\section{Corresponding Author:}

Anas A. Amaireh,

Telecommunications Engineering Department,

Yarmouk University,

P. O. Box 35, Irbid 21163, Jordan

Email: anasamaerih@yahoo.com; 2015976002@ses.yu.edu.jo

\section{INTRODUCTION}

The process of finding the optimal solution(s) for a particular problem is generally referred to optimization [1]. The main characteristic of the metaheuristic (stochastic) optimization algorithms is the randomness $[2,3]$, which means that random operators are employed in each iteration to find solutions close to the exact global optimum during the searching process. Therefore, in this kind of optimization there is no assurance to find the global optimum [4]. The wide use of stochastic optimization algorithms in the last two decades occurred due to four main reasons: simplicity, flexibility, local optima avoidance, and the needlessness of calculating the gradient of a solution.

Antenna is defined as a key part that wirelessly transmits or receives electromagnetic energy in any electronic system. According to certain specifications, the radiation of the antenna can be enhanced when numerous antennas are working together, which is defined as antenna array. The main feature of the antenna array compared to single antenna, is the ability to steer the main beam to transmit and receive electromagnetic waves without any mechanical movement for the array itself [5]. Antenna arrays are widely used in radar, satellite and mobile applications [6]. Several types and geometries have been studied in the literature, such as; linear, circular, elliptical, planar, ..., etc. The linear antenna array is the simplest and the most common type of antenna arrays [7].

In the literature, several stochastic optimization algorithms have been used for the synthesis of antenna arrays in order to get the desired radiation pattern, such as Firefly Algorithm (FA) [8], Particle Swarm Optimization (PSO) [9, 10], Ant Colony Optimization (ACO) [11], Taguchi Optimization [12], Invasive Weed Optimization (IWO) [13], Pattern Search optimization [14], Biogeography Based Optimization (BBO) [15], Antlion Optimization (ALO) algorithm [16] and Cat Swarm Optimization (CSO) [17]. In this work, two optimization algorithms are applied in the scanned antenna array problems; 
Antlion Optimization (ALO) algorithm [18] and new proposed hybrid optimization algorithm based on Grasshopper Optimization Algorithm (GOA) [19] and ALO.

The rest of this paper is organized as follows: In section 2, the proposed hybrid algorithm is presented. In section 3, the geometry and problem formulation for the scanned antenna arrays is presented. Then, the fitness function and the results with their discussion are presented in section 4 . Finally, the paper is concluded in section 5 .

\section{THE PROPOSED HYBRID OPTIMIZATION ALGORITHM}

\subsection{The theory of the hybrid method}

In [20-22], we proposed a new hybrid method that combines the features of two metaheuristic algorithms; Grasshopper Optimization Algorithm (GOA) [19] and Antlion Optimization (ALO) algorithm [18]. ALO is robust in exploitation process, which has been proved in many papers in the literature like $[23,24]$. The social forces in grasshopper's swarm show the strong ability in exploration process all over the search space. So, these features give the chance to combine these two methods, GOA and ALO, in a novel hybrid method, to make a huge enhancement on the performance of these algorithms. The benefits of hybridization can be represented in overcoming the main disadvantages of GOA and ALO. The full dependence on roulette wheel selection method is the main disadvantage in ALO, since it may cause; loss of diversity, early convergence and not enough pressure to select the fittest search agents among same fitness search agents. Whereas the main drawback of GOA exists in $c$ parameter equation, which weakens the exploitation process in the algorithm.

Our proposed Hybrid method can efficiently explore and exploit the whole search space to reach the global optimal value, because of merging the features of GOA and ALO together. The hybrid method has some factors that improve the ability of exploitation and exploration processes like: the population nature that decreases local optima stagnation, the attraction and repulsion forces in GOA method, the roulette wheel selection method and random walks in ALO method, choosing different samples of less and average fitness search agents from next position's matrix, and the modifications of $c$ parameter in GOA method. These factors and modifications lead to better diversity of the search agents all over the search space and high probability for local optima stagnation avoidance. Furthermore, the intensity of search agents in the proposed method has been reduced rapidly compared with ALO and GOA, due to the modification of $c$ parameter and its combination with other shrinking factors. Thus, the hybrid method guarantees the mature and fast convergence compared with ALO and GOA [22].

The following equations have been used in our proposed algorithms [22]:

$$
X_{i}^{d}=c\left(\sum_{\substack{j=1 \\ j \neq i}}^{N} c \frac{u b_{d}-l b_{d}}{2} s\left(\left|X_{j}^{d}-X_{i}^{d}\right|\right) \frac{X_{j}-X_{i}}{d_{i j}}\right)
$$

where $X_{i}^{d}$ defines the next position for $i^{\text {th }}$ grasshopper and $d^{\text {th }}$ dimension, $u b_{d}$ and $l b_{d}$ are the upper and lower bounds in the $d^{\text {th }}$ dimension, respectively, and $s(r)=f e^{\frac{-r}{l}}-e^{-r}$ where $f$ represents the intensity of attraction force and $l$ indicates the attractive length scale [22].

$$
\begin{aligned}
& \text { if } l \leq(L) / 2 \\
& c=\text { cmax }-l \frac{\text { cmax }-c m i n}{\left(\frac{L}{2}\right)} \\
& \text { if } l>\frac{L}{2} \\
& c=(L-l+1) \times\left(\frac{c \min }{L \times 10^{l}}\right)
\end{aligned}
$$

where $\operatorname{cmax}$ and $\mathrm{cmin}$ are the maximum and minimum values, respectively, $L$ indicates the maximum number of iterations, and $l$ is the current iteration [19].

$$
\begin{aligned}
& c^{t}=\frac{c^{t}}{I} \\
& d^{t}=\frac{d^{t}}{I}
\end{aligned}
$$


where $c^{t}$ and $d^{t}$ indicate the minimum and maximum of all variables at $t^{\text {th }}$ iteration, respectively, and $I$ represents a ratio, such that $I=10^{w} \frac{t}{T}$ where $t$ represents the current iteration, $T$ indicates the maximum number of iterations, and $w$ is a constant that depends on the current iteration [18].

\subsection{Flowchart of the hybrid algorithm [16]}

In Figure 1 show the flowchart of the hybrid algorithm [16].

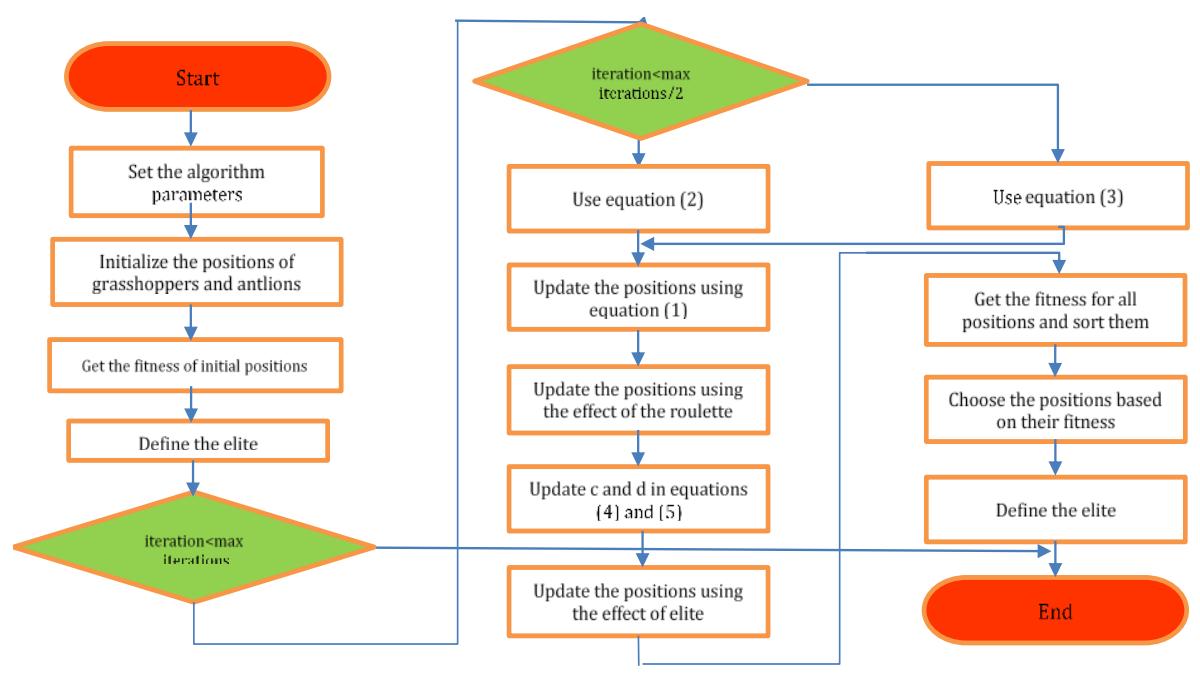

Figure 1. Flowchart of the hybrid algorithm

\section{GEOMETRY AND PROBLEM FORMULATION}

In scanned array antennas, the major lobe is steered to a specific direction, which can be applied in wide applications like mobile and cellular communications. To accomplish this, a progressive phase shift is added in the feeding currents [25]. Hence, the array factor equation for N-element scanned antenna array that lies along the $\mathrm{x}$-axis, with $\lambda / 2$ spacing between adjacent elements, can be written as follows [25]:

$$
A F(\varphi)=\sum_{n=1}^{N} I_{n} \exp \left(j \pi(n-1)\left[\cos (\varphi)-\cos \left(\varphi_{d}\right)\right]\right)
$$

where $I_{n}$ represents the excitation amplitude of the $n^{\text {th }}$ element. $\varphi_{d}$ is the angle at which the major lobe is steered. The geometry of such a scanned array with $\mathrm{N}$ elements is shown in Figure 2.

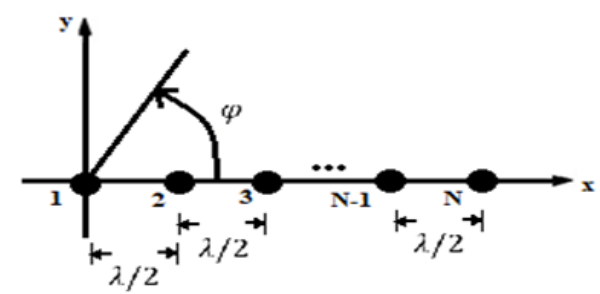

Figure 2. Geometry of N-element linear array

The main objective in this paper is to minimize the maximum side lobe level (SLL) with respect to the main lobe. With the purpose of accomplishing this goal, the following fitness function is minimized [24]:

$$
\begin{aligned}
& \text { fitness }=\max \left\{20 \log _{10}\left|\frac{A F(\varphi)}{A F\left(\varphi_{d}\right)}\right|\right\} \\
& \text { subject to } \varphi \in\left[0, \varphi_{s}\right]
\end{aligned}
$$

where $\varphi_{d}$ is the angle in the direction of the major beam. $\left[0, \varphi_{s}\right]$ represents the side lobes region that depends on the total number of the elements. 


\section{RESULTS AND ANALYSIS}

In this paper, three different examples are considered; 20-element LAA with $\varphi_{d}=30^{\circ}$, 26-element LAA with $\varphi_{d}=45^{\circ}$ and 30-element LAA with $\varphi_{d}=60^{\circ}$. In all the examples, the excitation currents are optimized to minimize the side lobe level using ALO and the proposed hybrid algorithm compared to Symbiotic Organisms Search (SOS) algorithm [25] and Firefly Algorithm (FA) [8] results. 40 search agents and 1000 iterations are used in all examples.

\subsection{Example 1: Optimization of 20-Element LAA $\left(\varphi_{d}=30^{\circ}\right)$}

In this example, the excitation currents for 20-element LAA with $\varphi_{d}=30^{\circ}$ have been optimized. Table 1 shows the optimal values for the feeding current amplitudes and the maximum SLL for ALO, the hybrid method, Symbiotic Organisms Search (SOS) [25] and Firefly Algorithm (FA) [8]. The maximum SLL values in $(\mathrm{dB})$ are $-15.45,-15.66,-15.64$ and -15.59 , respectively, for ALO, the hybrid method, SOS and FA techniques. Furthermore, the FNBW values are $29.56^{\circ}, 29.44^{\circ}, 29.44^{\circ}$ and $30.08^{\circ}$ for ALO, the hybrid, SOS and FA, respectively, and these are almost equal. The obtained radiation patterns and convergence curves for the proposed algorithms are displayed in Figure 3 and Figure 4. The statistical results for ALO and the hybrid method are tabulated in Table 2. It can be noticed that the standard deviation (STD) for both methods outperforms the STD for SOS and FA [25], which proves the stability and robustness of the proposed methods.

Table 1. Optimum currents for $30^{\circ}$ scanned LAA with 20 elements using ALO and the hybrid algorithm compared to other optimization techniques

\begin{tabular}{|c|c|c|c|}
\hline$\varphi_{d}=30^{\circ}, \mathrm{N}=20$ & Feeding current amplitudes $\left(I_{1} \ldots I_{20}\right)$ & Max. SLL (dB) & FNBW (degree) \\
\hline $\mathrm{ALO}$ & $\begin{array}{c}{[1.0000,0.48938,0.583,0.43543,0.25664,0.8691,0.48552,} \\
0.80474,0.3192,0.69249,0.5965,0.81911,0.7401,0.46723, \\
0.25881,0.79585,0.5334,0.23493,0.57805,0.99917]\end{array}$ & -15.45 & 29.56 \\
\hline Hybrid & $\begin{array}{c}{[0.86058,0.39071,0.31623,0.47141,0.36107,0.42318,} \\
0.59255,0.52504,0.43337,0.39471,0.64721,0.61082,0.49428, \\
0.40356,0.45944,0.47877,0.3173,0.41312,0.22736,1.0000]\end{array}$ & -15.66 & 29.44 \\
\hline SOS [25] & $\begin{array}{c}{[1.0000,0.2762,0.4499,0.3040,0.3787,0.6113,0.5305,0.5042,} \\
0.5554,0.6113,0.4950,0.4909,0.5940,0.4393,0.3429,0.5587, \\
0.4266,0.3142,0.4099,0.9092]\end{array}$ & -15.64 & 29.44 \\
\hline FA [8] & $\begin{array}{c}{[0.98041,0.76626,0.36907,0.55297,0.90715,0.20192,} \\
0.51964,0.84496,0.50948,0.98057,0.51426,0.53871,0.80273, \\
0.55406,0.88082,0.40378,0.33214,0.46556,0.50348,0.94604]\end{array}$ & -15.59 & 30.08 \\
\hline
\end{tabular}

Table 2. Performance of ALO and the hybrid algorithm for 20 independent runs

\begin{tabular}{lll}
\hline & ALO & Hybrid \\
\hline Min value $(\mathrm{dB})$ & -15.4466 & -15.6623 \\
Max value $(\mathrm{dB})$ & -15.1441 & -15.4003 \\
Median $(\mathrm{dB})$ & -15.3217 & -15.5586 \\
Mean $(\mathrm{dB})$ & -15.3087 & -15.5624 \\
STD $(\mathrm{dB})$ & 0.0859 & 0.0674 \\
\hline
\end{tabular}

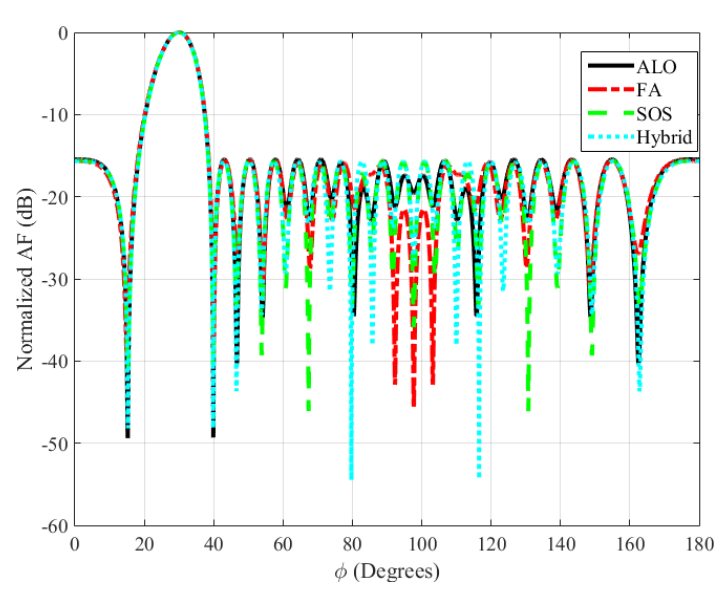

Figure 3. Radiation patterns of ALO and the hybrid technique for 20-element scanned LAA

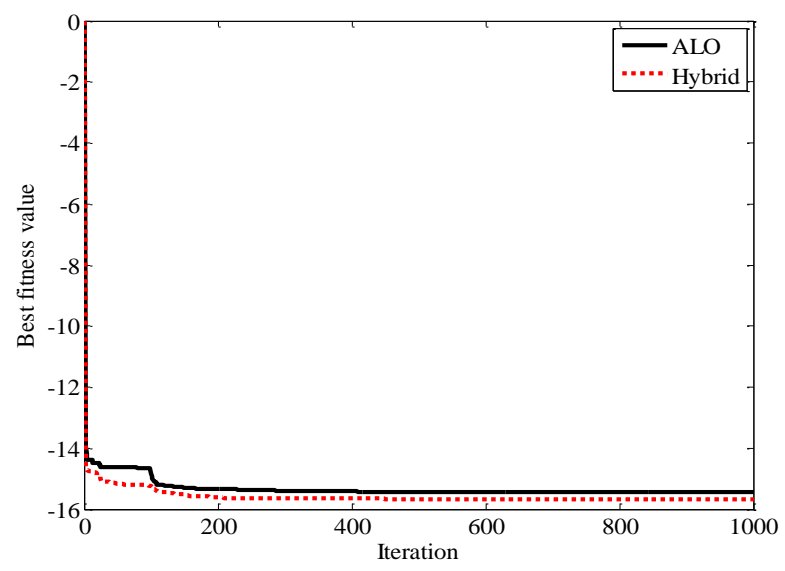

Figure 4. Convergence curves for the best result of scanned LAA using ALO and the hybrid algorithm 


\subsection{Example 2: Optimization of 26-Element LAA $\left(\varphi_{d}=45^{\circ}\right)$}

The optimum currents values and maximum SLL for 26-element $45^{\circ}$ scanned array antenna are tabulated in Table 3, while Table 4 shows the statistical properties for ALO and the hybrid method in 20 independent runs. Figure 5 and Figure 6 demonstrate the radiation patterns and convergence curves, respectively. It is obvious that the maximum SLL value obtained using the hybrid algorithm is slightly better than the ALO, SOS and FA techniques. For all methods, the FNBW=12.62.

Table 3. Optimum currents for $45^{\circ}$ scanned LAA with 26-element array using ALO and the hybrid method compared to other optimization techniques

\begin{tabular}{|c|c|c|}
\hline$\varphi_{d}=45^{\circ}, \mathrm{N}=26$ & Feeding current amplitudes $\left(I_{1} \ldots I_{26}\right)$ & Max. SLL (dB) \\
\hline ALO & $\begin{array}{c}{[0.9845,0.92464,0.010525,0.6751,0.62702,0.063028,0.65201,0.54108,} \\
0.5882,0.75892,0.49313,0.69067,0.82217,0.44326,0.56542,0.62967,0.65334, \\
0.53038,0.4111,0.7564,0.37021,0.74546,0.0055742,0.74332,0.36553,1.0000]\end{array}$ & -16.05 \\
\hline Hybrid & $\begin{array}{c}{[0.9777,0.34246,0.54211,0.084948,0.283,0.52902,0.52469,0.40156,0.45987,} \\
0.58482,0.35575,0.5388,0.53935,0.58524,0.38648,0.63499,0.47567,0.50461, \\
0.36721,0.45082,0.35472,0.3458,0.5698,0.23679,0.28481,1.0000]\end{array}$ & -16.32 \\
\hline SOS [25] & $\begin{array}{c}{[1.0000,0.2314,0.4243,0.4349,0.3933,0.4423,0.4890,0.3892,0.5260,0.5470} \\
0.3889,0.8891,0.4148,0.5557,0.4317,0.7241,0.4748,0.3302,0.7278,0.5896 \\
0.2174,0.5061,0.1908,0.4341,0.6199,0.9584]\end{array}$ & -16.18 \\
\hline FA [8] & $\begin{array}{c}{[1,0.72429,0.55905,0.44837,0.71979,0.31947,0.70758,0.62037,0.53995} \\
0.86304,0.67322,0.71588,0.83498,0.77957,0.42717,0.79538,0.71365 \\
0.63019,0.62672,0.6301,0.74732,0.060126,0.73871,0.59844,0.77826,0.9975]\end{array}$ & -15.61 \\
\hline
\end{tabular}

Table 4. Performance of ALO and the hybrid algorithm for 20 independent runs

\begin{tabular}{lll}
\hline & ALO & Hybrid \\
\hline Min value $(\mathrm{dB})$ & -16.0487 & -16.3188 \\
Max value $(\mathrm{dB})$ & -15.7675 & -16.1154 \\
Median $(\mathrm{dB})$ & -15.8969 & -16.2159 \\
Mean $(\mathrm{dB})$ & -15.9033 & -16.2146 \\
STD $(\mathrm{dB})$ & 0.0943 & 0.0911 \\
\hline
\end{tabular}

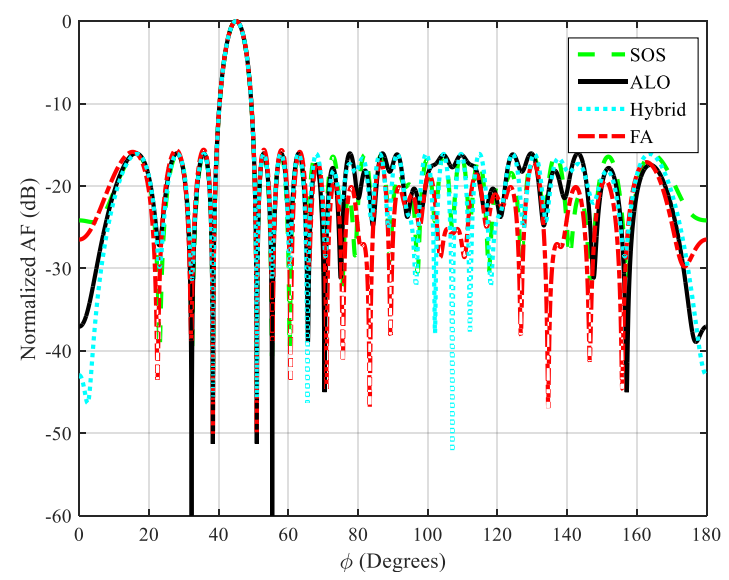

Figure 5. Radiation patterns of ALO and the hybrid technique for 26 elements scanned LAA

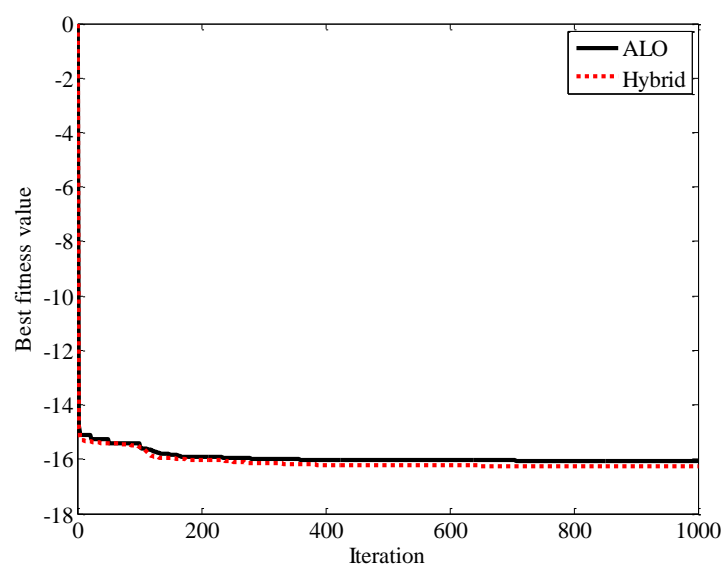

Figure 6. Convergence curves for the best result of scanned LAA using ALO and the hybrid algorithm

\subsection{Example 3: Optimization of 30-Element LAA $\left(\varphi_{d}=60^{\circ}\right)$}

The excitation currents for 30 elements in $60^{\circ}$ scanned antenna array have been investigated in this example. Table 5 shows the best value of the maximum SLL and FNBW values for ALO, the hybrid method, SOS and FA techniques. According to this table, it can be concluded that the hybrid algorithm somewhat beats other techniques. Table 6 mentions the consistency for ALO and the hybrid algorithm. The radiation patterns and convergence curves are illustrated in Figure 7 and Figure 8, respectively. 
Table 5. Optimum currents for $60^{\circ}$ scanned LAA with 30-element array using ALO and the hybrid method compared to other optimization techniques

\begin{tabular}{|c|c|c|c|}
\hline$\varphi_{d}=60^{\circ}, \mathrm{N}=30$ & Feeding current amplitudes $\left(I_{1} \ldots I_{30}\right)$ & Max. SLL (dB) & FNBW (degree) \\
\hline ALO & $\begin{array}{c}{[0.9380,0.5472,0.4980,0.4647,0.4777,0.0382,0.4824,0.7979,0.3462} \\
0.4455,0.6926,0.3170,0.6597,0.6022,0.7500,0.1379,0.8532,0.5132 \\
0.7009,0.1911,0.7336,0.7231,0.0303,0.6409,0.5290,0.3575,0.3012 \\
0.2129,0.7843,1.0000]\end{array}$ & -15.94 & 9 \\
\hline Hybrid & $\begin{array}{c}{[0.76681,0.31841,0.2907,0.33733,0.20298,0.30963,0.25103,0.4666} \\
0.33325,0.28434,0.2275,0.55575,0.41659,0.41468,0.35724,0.48412, \\
0.41547,0.27134,0.29311,0.47269,0.52282,0.29816,0.28305,0.35377 \\
0.32292,0.23532,0.34489,0.14076,0.23816,1.0000]\end{array}$ & -16.20 & 8.96 \\
\hline SOS [25] & 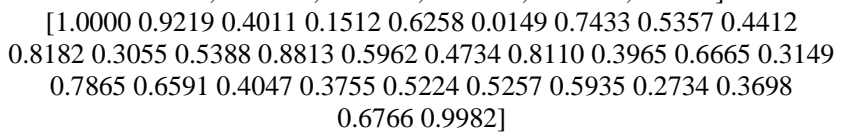 & -15.93 & 9 \\
\hline FA [8] & $\begin{array}{c}{[0.99575,0.68442,0.62997,0.049937,0.17937,0.73457,0.48525,} \\
0.61814,0.33365,0.63189,0.63643,0.39343,0.49183,0.77247,0.64547, \\
0.48407,0.73964,0.74411,0.52797,0.45014,0.82218,0.52901,0.45825, \\
0.41905,0.48686,0.24166,0.86684,0.63618,0.29691,0.99934]\end{array}$ & -15.97 & 9.08 \\
\hline
\end{tabular}

Table 6. Performance of ALO and the hybrid algorithm for 20 independent runs

\begin{tabular}{lll}
\hline & ALO & Hybrid \\
\hline Min value $(\mathrm{dB})$ & -15.9449 & -16.1959 \\
Max value $(\mathrm{dB})$ & -15.6637 & -15.9615 \\
Median value $(\mathrm{dB})$ & -15.8149 & -16.0773 \\
Mean value $(\mathrm{dB})$ & -15.8074 & -16.0822 \\
STD $(\mathrm{dB})$ & 0.0865 & 0.0501 \\
\hline
\end{tabular}

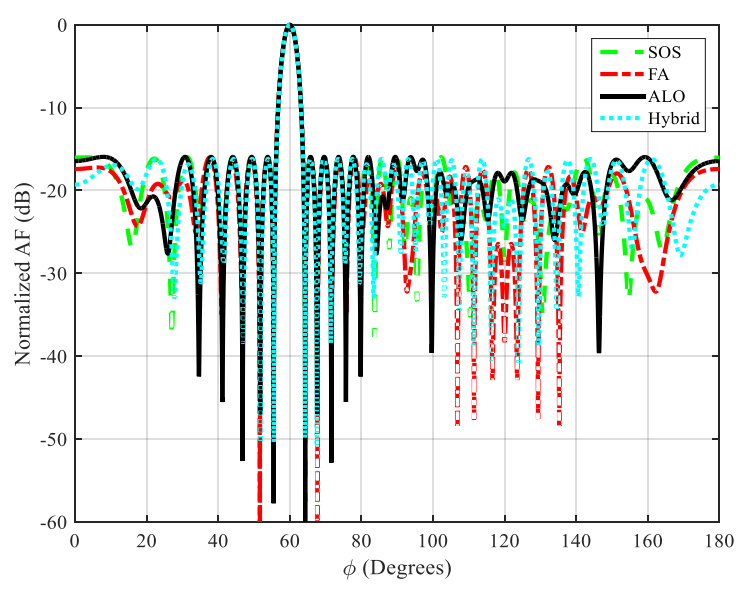

Figure 7. Radiation patterns of ALO and the hybrid technique for 30-element array scanned LAA

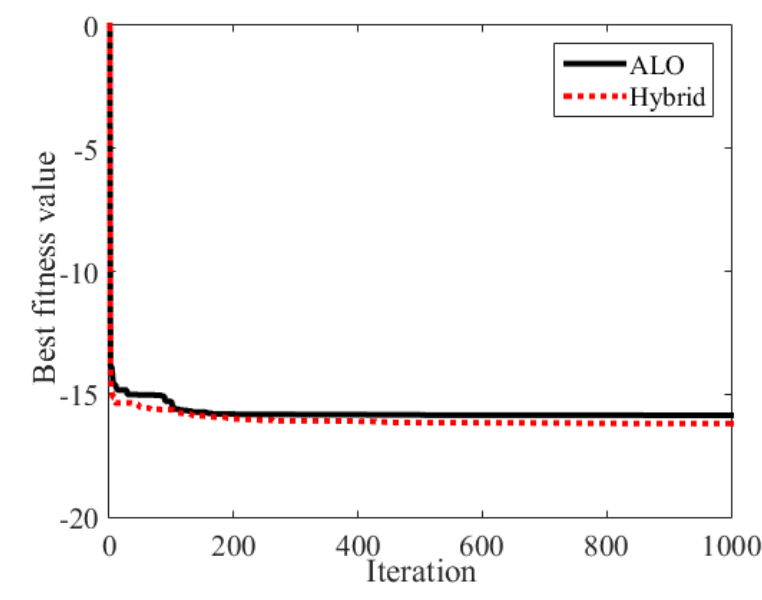

Figure 8. Convergence curves for the best result of scanned LAA using ALO and the hybrid algorith

\section{CONCLUSION}

In this paper, optimal design of scanned linear antenna arrays was performed using two metaheuristic algorithms; Antlion optimization (ALO) algorithm and our new proposed hybrid algorithm based on Grasshopper Optimization algorithm (GOA) and ALO. Our objective function in this paper was to reduce the side lobe level with the constraint of a fixed major lobe beamwidth. In this paper, three examples were discussed; 20-element, 26-element and 30-element scanned antenna array. The results show that the proposed hybrid algorithm is very competitive in reducing the SLL compared to other methods like; SOS and FA. In all experiments, a population size of 40 and 200 iterations only were enough to reach to the solution. 


\section{REFERENCES}

[1] S. Mirjalili, "Moth-flame optimization algorithm: A novel nature-inspired heuristic paradigm," Knowledge-Based Systems, vol. 89, pp. 228-249, 2015.

[2] J. C. Spall, Introduction to stochastic search and optimization: estimation, simulation, and control, John Wiley \& Sons, 2003.

[3] H. Hoos, T. Stützle, Stochastic local search: foundations \& applications, Elsevier, 2004.

[4] El-Ghazali Talbi, Metaheuristics: From Design to Implementation, Wiley \& Sons, 2009

[5] D. K. Cheng, "Optimization techniques for antenna arrays," in Proceedings of the IEEE, vol. 59, no. 12, pp. 16641674, Dec. 1971.

[6] S. An, S. Yang and Z. Ren, "Incorporating Light Beam Search in a Vector Normal Boundary Intersection Method for Linear Antenna Array Optimization," in IEEE Transactions on Magnetics, vol. 53, no. 6, pp. 1-4, June 2017, Art no. 7001304.

[7] A. Sharaqa, Biogeography-Based optimization method and its application in electromagnetics, Master thesis, Jordan University for Science and Technology, 2012.

[8] B. Basu, G. Mahanti, "Fire Fly and Artificial Bees Colony Algorithm for Synthesis of Scanned and Broadside Linear Array Antenna," Progress In Electromagnetics Research B, vol. 32, pp. 169-190, 2011.

[9] M. Bataineh, J. Ababneh, "Synthesis of aperiodic linear phased antenna arrays using particle swarm optimization," Electromagnetics, vol. 26, no 7, pp. 531-541, 2006.

[10] M. Shihab, Y. Najjar, N. Dib, M. Khodier, "Design of non-uniform circular antenna arrays using particle swarm optimization," Journal of Electrical Engineering, vol. 59, pp. 216-220, 2008.

[11] E. Rajo-Iglesias and O. Quevedo-Teruel, "Linear array synthesis using an ant-colony-optimization-based algorithm," in IEEE Antennas and Propagation Magazine, vol. 49, no. 2, pp. 70-79, April 2007.

[12] N. Dib, S. Goudos, H. Muhsen, "Application of Taguchi's Optimization Method and Self-Adaptive Differential Evolution to the Synthesis of Linear Antenna Arrays," Progress in Electromagnetics Research, vol. 102, pp. 159$180,2010$.

[13] S. Karimkashi and A. A. Kishk, "Invasive Weed Optimization and its Features in Electromagnetics," in IEEE Transactions on Antennas and Propagation, vol. 58, no. 4, pp. 1269-1278, April 2010.

[14] F. Gunes, F. Tokan, "Pattern search optimization with applications on synthesis of linear antenna arrays," Expert Systems with Applications, vol. 37, pp. 4698-4705, 2010.

[15] A. Sharaqa and N. Dib, "Design of linear and circular antenna arrays using biogeography based optimization," 2011 IEEE Jordan Conference on Applied Electrical Engineering and Computing Technologies (AEECT), Amman, 2011, pp. 1-6.

[16] A. Amaireh, N. Dib, A. Al-Zoubi, "The Optimal Synthesis of Concentric Elliptical Antenna Arrays," International Journal of Electronics, 2019, DOI: 10.1080/00207217.2019.1661028

[17] L. Pappula, D. Ghosh, "Linear antenna array synthesis using cat swarm optimization," International Journal of Electronics and Communications, vol. 68, pp. 540-549, 2014

[18] S. Mirjalili, "The Ant Lion Optimizer," Advances in Engineering Software, vol. 83, pp. 80-98, 2015.

[19] S. Saremi, S. Mirjalili, A. Lewis, "Grasshopper Optimisation Algorithm: Theory and application," Advances in Engineering Software, vol. 105, pp. 30-47, 2017.

[20] A. Amaireh, A. Al-Zoubi, N. Dib, "A New Hybrid Optimization Technique Based on Antlion and Grasshopper Optimization Algorithms," Submitted to Royal Society Open Science, 2019.

[21] A. Amaireh, Comparative Study of New Optimization Methods and Their Applications in Electromagnetics, Irbid: Master Thesis, Yarmouk University, 2018.

[22] A. Amaireh, A. Al-Zoubi, N. Dib, "Sidelobe-Level Suppression for Circular Antenna Array via New Hybrid Optimization Algorithm Based on Antlion and Grasshopper Optimization Algorithms," Progress In Electromagnetics Research C, vol. 93, pp. 49-63, 2019.

[23] N. Dib, A. Amaireh, A. Al-Zoubi, "On the Optimal Synthesis of Elliptical Antenna Arrays," International Journal of Electronics, vol. 106, no 1, pp. 121-133, 2019.

[24] A. A. Amaireh, A. Alzoubi and N. I. Dib, "Design of linear antenna arrays using antlion and grasshopper optimization algorithms," 2017 IEEE Jordan Conference on Applied Electrical Engineering and Computing Technologies (AEECT), Aqaba, 2017, pp. 1-6.

[25] N. Dib, "Design of Linear Antenna Arrays with Low Side Lobes Level Using Symbiotic Organisms Search," Progress In Electromagnetics Research B, vol. 68, pp. 55-71, 2016.

\section{BIOGRAPHIES OF AUTHORS}

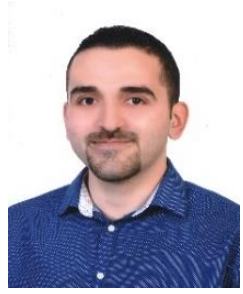

Anas Atef Amaireh received his B.Sc. and M.Sc. degrees in Engineering Technology with the major of Wireless Communication Engineering from Yarmouk University, Jordan in 2015 and 2018, respectively. His research interests are in antenna arrays, numerical techniques in electromagnetics, stochastic algorithms and optimization techniques. 


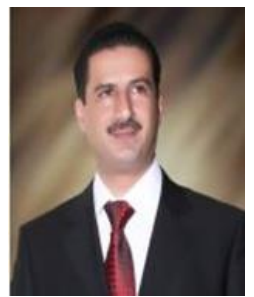

Asem S. Al-Zoubi received his B.Sc. degree in Electrical Engineering from Eastern Mediterranean University, Cyprus in 1993, the M.Sc. degree in Electrical Engineering from Jordan University of Science and Technology, Jordan in 1998, and the Ph.D. degree in Electrical Engineering/ Electromagnetics from the University of Mississippi, USA, in 2008. Currently, he is an Associate Professor with the Department of Telecommunications Engineering in Yarmouk University, Jordan. His current research interests include dielectric resonator antennas, microstrip antennas, and numerical techniques in electromagnetics. Mr. Al-Zoubi is a member of the IEEE

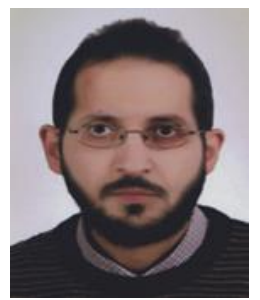

Nihad I. Dib obtained his B. Sc. and M.Sc. in Electrical Engineering from Kuwait University in 1985 and 1987, respectively. He obtained his Ph.D. in EE (major in Electromagnetics) in 1992 from University of Michigan, Ann Arbor. Then, he worked as an assistant research scientist in the radiation laboratory at the same school. In Sep. 1995, he joined the EE department at Jordan University of Science and Technology (JUST) as an assistant professor, and became a full professor in Aug. 2006. His research interests are in computational electromagnetics, antennas and modeling of planar microwave circuits 